\title{
Rothiamucilaginosa life threatening infections in non-neutropenic hosts
}

\author{
Graciela Faiad $^{1}$, Manmeet Singh ${ }^{1 *}$, Aarthi Narasimhan ${ }^{1}$, Marisa Mendez ${ }^{2}$, Shobha Sharma ${ }^{1}$, \\ Naiel Nassar ${ }^{1}$ \\ ${ }^{1}$ Infectious Disease Section, Department of Internal Medicine, University of California San Francisco-MEP Fresno, San Francisco, \\ USA; \\ ${ }^{2}$ Department of Pharmacy Services, University of California San Francisco-MEP Fresno, San Francisco, USA. \\ Email: msingh2@fresno.ucsf.edu
}

Received 18 October 2011; revised 5 November 2011; accepted 18 October 2011.

\begin{abstract}
Rothiamucilaginosa, previously known as Stomatococcus mucilaginous, resides in the oral cavity and respiratory tract as part of the normal flora [1]. It is a gram-positive, cocus-shaped bacterium. The bacterium is considered an emerging opportunistic pathogen in patients with chronic immunosuppressive diseases. Most of these reports were described in severe neutropenia or hematological cancer patients but less frequently in immune-competent hosts. We report two cases of endocarditis and meningitis due to $R$. mucilaginosain non-neutropenic hosts.
\end{abstract}

Keywords: Rothiamucilaginosa; Gram Positive; Neutropenia; Endocarditis; Meningitis; Opportunistic Infections

\section{INTRODUCTION}

Rothiamucilaginosa, previously known as Stomatococcus mucilaginous, resides in the oral cavity and respiratory tract as part of the normal flora [1]. It is a gram-positive, cocus-shaped bacterium. The bacterium is considered an emerging opportunistic pathogen in patients with chronic immunosuppressive diseases; and has been associated with endocarditis, meningitis, peritonitis, septicemia, osteomyelitis, late prosthetic joint infections and pneumonia in various case reports [1-3]. Most of these reports were described in severe neutropenia or hematological cancer patients but less frequently in immune-competent hosts. We report two cases of endocarditis and meningitis due to $R$. mucilaginosain non-neutropenic hosts.

\section{CASE REPORTS}

Patient one: A 52 year-old female former intravenous drug user, hospitalized with dyspnea on exertion, chest tightness, and confusion that started two days prior to admission. Her past medical history was significant for mitral and aortic infective endocarditis resulting in bioprosthetic replacement of both valves in 2004. On physical examination, the patient was afebrile, with poor dentition and splinter hemorrhages in two of her fingernails. Heart auscultation revealed a mid-systolic murmur with a click. Neurological exam was non focal. Laboratory tests only showed a white blood cell (WBC) count of 14,400 cells/ $\mu \mathrm{L}$. Blood cultures grew gram-positive cocci in clusters. The patient was started on intravenous vancomycin 1 gram, gentamicin $50 \mathrm{mg}$ every 12 hours and oral rifampin $300 \mathrm{mg}$ three times a day for empiric treatment of prosthetic valve endocarditis. This regimen was changed to intravenous penicillin $\mathrm{G} 2$ millions units every 4 hours and rifampin same dose once final identification of $R$. mucilaginosa was made by DNA sequencing at a reference lab. A transesophageal echocardiogram showed an aortic valve mass consistent with vegetation, and a smaller mobile one on the atrial side of the mitral valve. Both prosthetic valves showed abnormal motion. Magnetic Resonance Imaging of her brain showed multiple small subcortical and pontine embolic infarcts. A cardiothoracic surgery consultation was obtained to consider valve replacement however the patient was deemed too unstable to undergo surgical intervention.

Despite the clearance of bacteremia after 48 hours, the patient's mentation continued to deteriorate, requiring endotracheal intubation for airway protection. Later she developed acute kidney injury with signs of left and right heart failure associated with hemodynamic instability.

She completed a six-week course of intravenous antibiotics however her clinical status continued to worsen. She became ventilator dependent and required a tracheostomy. Patient continued to be febrile, with persistent leukocytosis but no sources of infection were identified. A transthoracic echocardiogram showed rocking and dehiscence of both bioprosthetic valves with evidence of a periaortic abscess and severe pulmonary hypertension. 
At that time cardiothoracic surgery was consulted and the patient was deemed to be a non-surgical candidate due to her multiple co-morbidities. A second course of antibiotics was started with intravenous ampicillin 2 grams every 4 hours and rifampin 300mg every 8 hours. After two weeks of therapy patient continued to do poorly, never recovering her baseline mental status and subsequently she was placed on comfort care as per family wishes.

Patient two: A 21 years old male presented with fevers, headache, multiple falls and altered mental status. Two years prior to admission he was diagnosed with Pre-B acute lymphocytic leukemia. At that time an Omaya reservoir was placed and he underwent intravenous and intrathecal administration of chemotherapy with subsequent remission. Seven weeks prior to this admission he noted fatigue, subjective fevers and generalized weakness. A bone marrow biopsy showed a relapse of his disease and he was treated with a second course of chemotherapy that was completed three weeks prior to admission. Since then, the patient continued to have intermittent fevers and a severe headache that could not be controlled with analgesics. On admission the patient was febrile, awake and alert, slightly confused, with a bulging Omaya reservoir, but no other focal neurological abnormalities. Laboratory tests showed WBC count of 2.100 cells $\mu / \mathrm{L}$, with $31 \%$ lymphocytes and an absolute neutrophil count of 1364 cells $/ \mathrm{mm}^{3}$; a hemoglobin concentration of $10.6 \mathrm{~g} / \mathrm{dL}$ and a normal platelet count. A CSF specimen obtained from the Omaya reservoir revealed an elevated leukocyte count (198 cells $/ \mathrm{mm}^{3}$ ) with 95\% PMNs. Gram stain examination revealed the presence of gram-positive cocci in clusters. Empiric therapy was started with intravenous vancomycin 1.5 grams and ceftriaxone 2 grams twice daily. A brain CT showed hydrocephalus involving the lateral ventricles. Twentyfour hours later the patient underwent removal of the Omaya reservoir and placement of an external ventricular drain (EVD) for management of the hydrocephalus and administration of intra-thecalvancomycin. Once final identification of $R$. mucilaginosa was confirmed, rifampin was added to the previous regimen, and ceftriaxone was discontinued. The patient received intravenous (IV) vancomycin with dose adjusted to renal function and to maintain a serum trough of $15-20 \mu \mathrm{g} / \mathrm{ml}$ in addition to oral rifampin $600 \mathrm{mg}$ daily for a total of 2 weeks. The patient also received $10 \mathrm{mg}$ of intra-thecal (IT) vancomycin for 5 days. His symptoms resolved over the fourteen-day hospital stay, his EVD was removed and he was discharged home. One month later he was admitted for placement of a ventriculoperitoneal shunt due to development of confusion with obstructive hydrocephalus. All CSF cultures remained negative. Patient was later discharged without complications.

\section{MICROBIOLOGY}

The organism was identified after initial growth in blood culture bottles incubated under aerobic and anaerobic conditions using the BacT/ALERT system.

Blood gram stain showed gram-positive cocci in clusters. Subcultures to sheep blood agar, chocolate agar and macconkey agar were incubated aerobically. Subculture on brucella agar was anaerobically incutabed. The organism formed $2 \mathrm{~mm}$, non-hemolytic whitish colonies that became adherent to blood agar after 72 hours of incubation.

\section{DISCUSSION}

$R$. mucilaginosa can cause opportunist infections in neutropenic, immune-compromised and hematological malignancies patients [1-3]. Infections due to this commonly believed low virulence organism, have been rarely reported in non-neutropenic patients and always has been associated with pre-existing risk factors. These include profound immune-compromise states, prophylactic antibiotic use, repeated broad-spectrum antibiotics exposure, mucositis, injectable drug use, abnormal cardiac valves, and chronic indwelling catheter infections [4-11]. Taxonomic confusion and misidentification with the common skin contaminants like staphylococcus, streptococcus or micrococcus, can lead to serious complications and delays in treatment.

Medical literature review revealed only seven well-

Table 1. Biochemical characteristics of R. mucilaginosaisolated from bloodstream and spinal fluid.

\begin{tabular}{cc}
\hline Biochemical Test & Reaction \\
\hline Blood Agar & Positive \\
Chocolate Agar & Positive \\
Bile Esculin Agar & Negative \\
PYR Test & Positive \\
\hline
\end{tabular}

Table 2. Broth dilution MICs for $R$. mucilaginosaisolated from the bloodstream and spinal fluid.

\begin{tabular}{ccc}
\hline Antibiotics & $\begin{array}{c}\text { MIC (mg/liter) } \\
\text { (Blood) }\end{array}$ & $\begin{array}{c}\text { Susceptibilities (mg/liter) } \\
\text { (spinal fluid) }\end{array}$ \\
\hline Penicillin & 0.12 & 0.5 \\
Rifampin & $<0.5$ & - \\
Vancomycin & 1.0 & 1.0 \\
Linezolid & 0.5 & - \\
Gentamicin & 1.0 & - \\
Daptomycin & 4.0 & - \\
Quinupristin/Dalfopristin & $<0.5$ & - \\
\hline
\end{tabular}


documented cases of $R$. mucilaginosa endocarditis [811]. Of interest, all reported cases involved mitral and aortic valves only whereas tricuspid and pulmonic valves were spared. Rothia can affect both native and prosthetic valves. As previously noted that relatively noninvasive organisms can cause left sided endocarditis in intravenous drug users most often in the setting of previously damaged or replaced valves. In this respect, $R$. mucilaginosa may behave in a fashion similar tootherorganisms of low virulence that may contaminate the skin, gain entry to the blood stream during non-sterile venipuncture, and colonize altered valve surfaces by virtue of prominent adherence properties [11].

These reported Rothia endocarditis cases uniformly shows antimicrobial susceptibility to penicillin and vancomycin including one case treated in combination with gentamicin and rifampin [10]. All cases, except for 2 (including our reported case) recovered with medical or conjoint medical/surgical therapy. Mortality in this series was $25 \%$. Two fatalities were observed; one associated with the presence of a periaortic abscess and the other with a significantly large aortic vegetation associated with myocardial involvement [8-11]. Our case shares similarity with other reported cases as presented with left sided endocarditis in an intravenous drug user with bioprosthetic valves; and the isolate was susceptible to penicillin. One interesting observation is that involved both mitral and aortic valves; developed a periaortic abscess and septic embolism to the brain making the case fatal.

In terms of Rothia associated meningitis cases, vancomycin administered both intravenously and intrathecally showed better clinical outcomes and survival than using intravenous vancomycin alone [12-14].This may be explained by low CSF concentrations of intravenous vancomycin; and reported cases with intermediate susceptibility to vancomycin [14]. In our meningitis case the use of IV and IT vancomycin in addition to rifampin after reservoir removal, was curative [14].

There are no guidelines to assist in antibiotic selection in the treatment of invasive Rothia infections. Third generation cephalosporins, vancomycin, high dose ampicillin, rifampin and chloramphenicol are reported to be active [15]. Von Eiff et al. reported the in vitro activity of other agents including teicoplanin and fusidic acid, however those agents are not available in the US [15]. Penicillin used to be the drug of choice for Rothia infections [15]. Due to an increased number of reported cases of relative penicillin resistance, other agents are increasingly needed $[15,16]$.

\section{CONCLUSIONS}

No doubt the number of reported cases with $R$. mucilaginosa will continue to grow. Nonetheless, this organism should be recognized as having ability to cause serious infection not only in immune-compromised, neutropenic patients but also in relatively healthy individuals with associated artificial/damaged valves or indwelling catheters.

Finally some important points for successful treatment of $R$. mucilaginosainfection are its early and appropriate identification, initiation of appropriate empiric antibiotic therapy followed by directed antibiotic therapy according to susceptibility testing, and most importantly early removal of the infected device.

\section{REFERENCES}

[1] McWhinney, P.H., Kibbler, C.C., Gillespie, S.H., Patel, S., Morrison, D., Hoffbrand, A.V. and Prentice, H.G. (1992) Stomatococcusmucilaginosus: An emerging pathogen in neutropenic patients. Clinical Infectious Diseases, 14, 641-646. doi:10.1093/clinids/14.3.641

[2] Henwick, S., Koehler, M. and Patrick, C.C. (1993) Complications of bacteremia due to Stomatococcusmucilaginosus in neutropenic children. Clinical Infectious Diseases, 17, 667-671. doi:10.1093/clinids/17.4.667

[3] Gruson, D., Hilbert, G., Pigneux, A., Vargas, F., Guisset, O., Texier, J., Boiron, J.M., Reiffers, J., Gbikpi-Benissan, G. and Cardinaud, J.P. (1998) Severe infection caused by Stomatococcusmucilaginosus in a neutropenic patient: case report and review of the literature. Hematology and Cell Therapy, 40, 167-169.

[4] Van Tiel, F.H., Slangen, B.F., Schouten, H.C. and Jacobs, J.A. (1995) Study of Stomatococcusmucilaginosusiso lated in a hospital ward using phenotypic characterization. European Journal of Clinical Microbiology and Infectious Diseases, 14, 193-198. doi:10.1007/BF02310355

[5] Lee, A.B., Harkery-Murray, P., Ferrieri, P., Schleiss, M.R. and Tolar, J. (2008) Bacterial meningitis from rothiamucilaginosa in patients with malignancy or undergoing hematopoietic stem cell transplantation. Pediatric Blood Cancer, 50, 673-676. doi:10.1002/pbc.21286

[6] Mitchell, P.S., Huston, B.J., Jones, R.N., Holcomb, L. and Koontz, F.P. (1990) Stomatococcusmucilaginosusbacteraemias: Typical case presentations, simplified diagnostic criteria, and a literature review. Diagnostic Microbiology and Infectious Disease, 13, 521-525. doi:10.1016/0732-8893(90)90085-A

[7] Goldman, M., Chaudhary, U.B., Greist, A. and Fausel, C.A. (1998) Central nervous system infections due to Stomatococcusmucilaginosus in immunocompromised hosts. Clinical Infectious Diseases, 27, 1241-1246. doi:10.1086/515001

[8] Relrnan, D.A., Ruoff, K., Ferraro, M.J. (1987) Stomatococcusmucilaginosusendocarditis in an intravenous drugabuser. Journal of Infectious Diseases, 155, 1080-1082. doi:10.1093/infdis/155.5.1080

[9] Coudron, P.E., Markowitz, S.M., Mohanty, L.B., Schatzki, P.F., Payne, J.M. (1987) Isolation of Stomatococcusmucilaginosusfrom a drug user with endocarditis. Journal of Clinical Microbiology, 25, 1359-1363.

[10] Pinsky, R.L., Piscitelli, V. and Patterson, J.E. (1989) Endocarditis caused by relatively penicillin-resistant Stoma- 
tococcusmucilaginosus. Journal of Clinical Microbiology, 27, 215-221.

[11] Rubin, S.J., Lyons, R.W. and Murcia, A. J. (1978) Endocarditis associated with cardiac catheterization due to a gram-positive coccus designated Micrococcus mucilaginosusincertaesedis. Journal of Clinical Microbiology, 7, 546-549.

[12] Schaad, U.B., Nelson, J.D., McCracken, G.H. Jr. (1981) Pharmacology and efficacy of vancomycin for staphylococcal infections in children. Rev Infect Dis., 3, S282S288.

[13] Luer, M.S. and Hatton, J. (1993) Vancomycin administration into the cerebrospinal fluid: A review. Annals of Pharmacotherapy, 27, 912-921.
[14] Skogen, P.G., Kolmannskog, S. and Bergh, K. (2001) Bactericidal activity in cerebrospinal fluid by treating meningitis caused by Stomatococcusmucilaginosus with rifampicin, cefotaxime and vancomycin in a neutropenic child. Clinical Microbiology and Infection, 7, 39-42. doi:10.1046/j.1469-0691.2001.00182.x

[15] Von Eiff, C., Herman, M. and Peters, G. (1995) Antimicrobial susceptibilties of stomatococcus Mucilaginosa and of Micrococcus spp. Antimicrobial Agents and Chemotherapy, 39, 268-270.

[16] Kaiser, A.B. and McGee, Z.A. (1975) Aminoglycoside therapy of gram-negative bacillary meningitis. New England Journal of Medicine, 293, 1215-1220. doi:10.1056/NEJM197512112932401 